\title{
Le système éducatif cubain depuis la crise des
} années 1990

Philippe Bayart, Rémy Herrera et Éric Mulot

\section{OpenEdition}

12 Journals

Édition électronique

URL : http://journals.openedition.org/ries/511

DOI : 10.4000/ries.511

ISSN : 2261-4265

Éditeur

Centre international d'études pédagogiques

\section{Édition imprimée}

Date de publication : 1 septembre 2008

Pagination : 153-160

ISBN : 978-2-85420-573-2

ISSN : $1254-4590$

Référence électronique

Philippe Bayart, Rémy Herrera et Éric Mulot, « Le système éducatif cubain depuis la crise des années 1990 », Revue internationale d'éducation de Sèvres [En ligne], 48 | septembre 2008, mis en ligne le 28 juin 2011, consulté le 26 avril 2021. URL : http://journals.openedition.org/ries/511 ; DOI : https://doi.org/ $10.4000 /$ ries.511

Ce document a été généré automatiquement le 26 avril 2021

(c) Tous droits réservés 


\title{
Le système éducatif cubain depuis la crise des années 1990
}

\author{
Philippe Bayart, Rémy Herrera et Éric Mulot
}

Les études concernant l'éducation à Cuba sont peu connues puisqu'elles sont menées en dehors du cadre de l'OCDE, souvent source unique d'information pour les observateurs occidentaux. De récentes évaluations internationales (Unesco, CEPAL, PNUD, CIEM) ont montré que les résultats obtenus par le système éducatif cubain le placent largement en tête des pays d'Amérique latine. Dans certains domaines l'école cubaine dépasse maints pays développés, dont la France. Les expertises sur le terrain ont permis de constater le fonctionnement régulier et universel de l'école cubaine en dépit de son extrême pauvreté, conséquence de l'embargo imposé par les États-Unis.

Il faut savoir gré à Philippe Bayard, Rémy Herrara et Éric Mulot, tous chercheurs à l'université Paris 1, de fournir une analyse, fût-elle partiale, de ce système éducatif hors normes occidentales. L'article rappelle les principes sur lesquels est basée l'école cubaine, principes tendant à remplacer la conception capitaliste de la société par une organisation sociale orientée vers plus d'équité. L'école cubaine s'est effectivement fondée dès la révolution de 1959 sur l'éradication de l'analphabétisme particulièrement dans les zones rurales et le développement d'un système scolaire centralisé, gratuit, égal pour tous. La crise survenue en 1991, avec la disparition du COMECOM qui liait les différents pays du bloc communiste et assurait une aide substantielle à Cuba depuis 1972, a entraîné outre de graves tensions sociales liées à la pénurie, une baisse des taux de scolarisation et du niveau global, ainsi qu'une désaffection de la voie universitaire et de la fonction enseignante. Comment les dirigeants cubains tentent-ils de remédier à cette crise sans renoncer aux objectifs premiers, ni recourir à la libéralisation de l'éducation que connaissent tant de pays actuellement? Les auteurs de l'article apportent un regard neuf sur la politique éducative cubaine, ainsi qu'une information précieuse sur la situation dans «l'île rebelle ». Pierre-Louis Gauthier 
1 À Cuba, l'éducation constitue, on le sait, un axe essentiel du projet de développement et un moyen d'atteindre l'objectif d'égalité. L'une des finalités du système éducatif y est de remettre en cause la division capitaliste du travail et la division sociale qui en découle. Les principes fondamentaux qui orientent les politiques éducatives cubaines sont l'universalisme, la gratuité et le caractère public de l'éducation. Leur application a permis d'édifier l'un des meilleurs systèmes éducatifs au monde, en termes d'accès comme de qualité. La question qui nous occupera dans cet article sera de savoir dans quelle mesure la crise économique qui suivit la disparition du bloc soviétique a mis à mal le secteur éducatif à Cuba. Nous verrons que, si Cuba a su préserver les piliers de son système éducatif, d'importantes innovations sont intervenues depuis les années 1990.

\section{Effets de la crise sur l'éducation}

2 Au début des années 1990, l'économie cubaine traversa une crise gravissime, liée à la dislocation des échanges avec le COMECON et au durcissement de l'embargo imposé par les États-Unis. L'égalitarisme et l'homogénéité de la société cubaine n'en sont pas sortis intacts. La répartition égalitaire du revenu et l'existence d'un système complet de services publics ont constitué la réussite, comme aussi l'une des justifications du processus révolutionnaire. Mais ce dernier s'est trouvé déstabilisé par la dollarisation ${ }^{1}$. Pour la première fois depuis 1959 , les inégalités ont augmenté - sans toutefois atteindre celles observées en Amérique latine. À Cuba, la survie des catégories sociales les plus modestes n'a pas été menacée, car l'État a garanti un accès universel et (presque) gratuit à un panier de consommation (grâce à la libreta pour l'alimentation) et aux services sociaux ${ }^{2}$. La stratégie de recuperación mise en œuvre à partir de 1993-94, qui est passée par des réformes d'ouverture mesurée aux mécanismes de marché, sans retour au capitalisme, a atteint, pour partie, les objectifs qu'elle s'était fixée. Depuis 1995, l'économie s'est redressée, et la dédollarisation est engagée depuis 2004. La société cubaine, pourtant très choquée par la résurgence d'inégalités, ne s'est pas déchirée. Les bases du système social ont été ébranlés, mais sont restés debout. La priorité a continué d'être accordée aux dépenses sociales, au rôle contracyclique.

Les budgets consacrés au secteur éducatif se sont certes trouvés affectés par la contraction du PIB du début de la décennie 1990. Exprimées en valeur, les dépenses d'éducation n'ont retrouvé leur niveau de 1989 qu'en 1999. Mais la croissance de ces dépenses est redevenue positive dès 1994, pour s'accélérer après 1998. La proportion des budgets éducatifs dans le PIB s'est même accrue entre 1990 et 1993, alors que ce dernier chutait $(-35 \%)$. Les années 2000 se caractérisent par un rythme de progression de la part des dépenses d'éducation dans le PIB plus élevée que celle des autres dépenses sociales (respectivement $76 \%$ et $44 \%$ ). Le budget pour 2006, représentant plus de deux fois celui de 2000, traduit une nouvelle hausse des dépenses d'éducation. Cuba est donc parvenue à poursuivre son projet social, salué par nombre d'observateurs étrangers ${ }^{3}$, tout spécialement grâce au système éducatif. 


\section{La stratégie éducative cubaine}

4 Dans de nombreux rapports rédigés en collaboration avec des experts d'organisations internationales, des chercheurs et responsables de la politique éducative cubaine utilisent la terminologie forgée par ces dernières, en faisant référence, par exemple, aux notions d'équité ou de capital humain ${ }^{4}$. Le premier concept est cependant distinct de celui d'égalité prévalant à Cuba, tandis que le second est interprété différemment de la théorie du capital humain, en ce qu'il ne réduit pas le travail qualifié à un capital, mais favorise le développement intégral de la personne ${ }^{5}$. Cuba n'a cessé de rejeter la logique et la pratique de ces stratégies conçues par les experts internationaux, et continue d'affirmer les principes qui fondent ses propres politiques sociales. Le traitement préférentiel des strates démunies et marginales à Cuba s'écarte du ciblage car il correspond sur l'île à des assistances personnalisées venant renforcer une couverture universelle. L'usage du concept d'équité se fait à Cuba dans le cadre d'une réflexion de fond où l'idée d'« égalité des opportunités » n'est pertinente que pour autant qu'elle est une dimension de l'égalité des conditions. Les moyens préconisés par les organisations internationales pour la concrétiser sont rejetés. Une telle égalité implique en effet que chacun ait accès à un service éducatif de qualité identique. Réaliser simultanément l'égalité d'accès et de qualité ne pourrait se faire qu'à travers « un niveau de préparation homogène dans toutes les écoles, auxquelles ont accès tous les élèves, indépendamment du lieu de résidence ou de la situation familiale $»^{6}$. Seule la centralisation des programmes et des plans d'études, excluant la marchandisation de l'éducation, est susceptible de lutter contre les inégalités.

Quant à la décentralisation, le gouvernement cubain affirme avoir atteint le double objectif d'équité et de développement intégral grâce à l'application d'une politique sociale unique et dirigée par l'État. La position cubaine est qu'une différenciation de l'éducation selon les besoins entre en contradiction avec le principe d'équité, puisque ces différences viennent pour partie des conditions économiques et de la place occupée par les individus dans la division du travail. Il convient alors de garantir des " conditions éducatives similaires » et de rendre l'éducation effectivement obligatoire. Le caractère homogène de l'éducation est indispensable à l'équité, par la recherche d'équilibre entre centralisation et décentralisation, massification et qualité, unité et diversité. De plus, la participation à la réalisation des objectifs concerne directement les enseignants, invités, en fin d'année scolaire, à évaluer le travail accompli, à en identifier les problèmes et réfléchir ensemble aux moyens de les dépasser par des propositions transmises à l'administration centrale. Gestion des infrastructures et financement des objectifs planifiés sont centralisés, mais les informations et les suggestions émanant des unités locales sont largement diffusées et discutées. Cette organisation comporte des défectuosités, comme par exemple un risque de routinisation, mais elle offre des avantages, notamment pour la résolution des difficultés rencontrées au niveau local, la réalisation de l'équité et la gestion qualitative du système. Une auto-évaluation des enseignements a été mise en place, qui implique aussi les élèves. Ses résultats sont incorporés aux dispositifs de formation continue des enseignants et aux recherches pédagogiques menées par le ministère - auxquelles est convié un nombre croissant d'enseignants, pour un «perfectionnement continu » participatif. 


\section{Spécificités du système cubain et innovations pédagogiques récentes}

6 Les contraintes budgétaires n'ont entraîné aucune fermeture d'établissement scolaire, ni d'interruption de cours ou de détérioration sensible de la qualité des enseignements, ni même - à quelques exceptions près - de pénurie globale de professeurs, quel que soit les niveaux ${ }^{7}$. Le fondement démocratique du projet éducatif a été préservé, à savoir la gratuité d'accès. Cela ne signifie évidemment pas que le fonctionnement du secteur éducatif n'a pas été touché8. Pendant la crise, les problèmes de transports liés au manque de combustibles ont gêné la mobilité des étudiants. Des manques ont concerné les matériels pédagogiques et didactiques pour les classes et l'approvisionnement en produits alimentaires pour les cantines. L'embargo a entraîné des surcoûts à l'importation de matériels scolaires - élevés, par exemple, dans l'éducation spécialisée des enfants handicapés, sans que celle-ci, prioritaire, n'ait cessé de bénéficier de moyens importants (pour l'accès aux technologies de l'information [écran tactile, appareils à réponse vocale, claviers intelligents, machines en braille...] et l'intégration professionnelle). Il a fallu trouver parfois des solutions d'urgence : récupération de matériels et réparation d'écoles, remise en question de la spécialisation par discipline des professeurs et diversification des compétences, programmes de formation accélérée, déplacement de professeurs au domicile des élèves, mobilisation de toute la collectivité pour la protection et la formation de la petite enfance...

7 Mais en dépit de ces difficultés, l'universalisation de l'enseignement a pu être préservée, tant bien que mal, et l'homogénéisation des indicateurs est restée forte entre zones urbaine et rurale 9 . Le taux de scolarisation dans le primaire est toujours demeuré supérieur à $99 \%$ depuis 1990. Dans le secondaire, ce taux a baissé jusqu'en 1995, avant de se redresser et de dépasser dès 1998 son niveau du début de la décennie. Une recrudescence des abandons d'études fut toutefois enregistrée en 1994. L'évolution démographique ne rend compte que partiellement de ce phénomène, lequel s'explique surtout par l'accentuation des difficultés économiques et par une certaine inadaptation des formations proposées aux besoins du pays ${ }^{10}$. Le choix des élèves s'est porté sur des études professionnelles et techniques courtes. Les sections pré-universitaires ont été un temps délaissées au bénéfice de nouveaux Instituts polytechniques assurant des formations aux emplois des services (tourisme, comptabilité, informatique). Les efforts entrepris pour adapter davantage les enseignements aux attentes professionnelles des étudiants ont permis de réduire peu à peu les taux d'abandon.

Plusieurs réformes pédagogiques ont été engagées pour faire face aux nouveaux défis du secteur éducatif. Les innovations ont visé à améliorer la qualité des enseignements primaire et secondaire, en consolidant l'apprentissage des matières fondamentales (espagnol, histoire, mathématiques) par un accroissement du nombre d'heures qui leur sont consacrées, mais aussi en promouvant les formations culturelles et artistiques et les nouvelles technologies. Les écoles du pays sont désormais équipées de plus de 50000 ordinateurs, dont 24000 dans les écoles primaires. La "révolution éducative ", prolongée par la "bataille des idées", a privilégié la créativité et la participation pédagogiques pour atteindre une "culture générale intégrale ». Cet objectif a nécessité d'importants moyens en termes de supports didactiques et informatisés. Les formations à l'informatique sont aujourd'hui généralisées. En 2005, 850000 jeunes bénéficiaient des équipements du Réseau de Clubs d'Informatique. Ont été ouvertes des Écoles 
d'informatique, accueillant plus de 20000 élèves, et les nombreuses antennes de l'Université des Sciences informatiques contribuent à développer l'industrie du software éducatif. Aujourd'hui, du préscolaire au secondaire, toutes les classes disposent d'au moins un ordinateur et d'un équipement audiovisuel.

9 La télévision est devenue un outil important de diffusion, transmission et assimilation des connaissances. Une chaîne entièrement consacrée à l'enseignement (Canal Educativo) et émettant plus de quinze heures par jour, y compris le week end et la nuit, est désormais accessible à près de $90 \%$ de la population. L'une des chaînes de la télévision nationale retransmet aussi les programmes Universidad para Todos, proposant, sous la forme de "salles de classe", des enseignements de qualité en mathématiques, histoire, langues étrangères et arts. Ainsi, 30 \% des plannings télévisuels des chaînes cubaines - sans aucune publicité commerciale - sont au total consacrés à des émissions éducatives, en plus des programmes de Canal Educativo.

Ces réformes ont porté leurs fruits. Aujourd'hui, $100 \%$ des enfants cubains intègrent le $7^{e}$ degré et 99,5\% d'entre eux valident le $9^{e}$ degré. Cuba est devenu le pays disposant du plus grand nombre d'enseignants par habitant et du plus faible nombre d'élèves par classe dans les cycles primaire et secondaire : le ratio élèves par maître est de 19 dans le primaire et 15 dans le secondaire. Les résultats obtenus par Cuba lors d'évaluations comparatives effectuées dans le cadre des tests internationaux en constituent l'une des preuves les plus tangibles. À titre d'exemple, Cuba se classait à la première place d'un test régional de l'UNESCO en 1998, sur un échantillon des douze plus grands pays d'Amérique latine. Tel est le cas des tests passés par les élèves de $3^{\mathrm{e}}$ et $4^{\mathrm{e}}$ années d'école primaire en langue maternelle et mathématiques. Quel que soit le niveau, pour toutes les matières, la position de Cuba était "notablement supérieure " ${ }^{11}$ à celles des autres pays enquêtés (dont l'Argentine, le Chili, le Costa Rica, la Colombie, le Mexique, le Brésil...). En 2005, une étude conduite par Altinok, visant à homogénéiser les résultats obtenus lors des tests comparés de compétences éducatives à l'échelle mondiale, aboutit à la construction d'Indices qualitatifs de Capital humain (IQCH). Les classements établis placent Cuba en très bonne position, au-dessus de la plupart des pays "développés " ${ }^{12}$. La compilation de six enquêtes internationales, incluant 50000 enfants et cent classes par pays, révèle un IQCH de Cuba supérieur à celui de la France pour les mathématiques. Les évaluations de compétences en mathématiques (100 pays) et en langue maternelle (88 pays) classent Cuba aux dixièmes places. Dans ces classements, seuls le Canada, la Finlande, Hong Kong et la Corée du Sud dépassent Cuba. Ces enquêtes montrent, que, malgré les difficultés rencontrées, les choix opérés par le secteur éducatif cubain ont dans l'ensemble été efficaces. Les problèmes ont été surmontés sans remise en cause du caractère universel, gratuit et public de l'éducation.

\section{Éducation supérieure et emploi}

11 L'effet négatif le plus visible de la crise sur l'éducation s'est manifesté par une baisse très sensible du nombre d'étudiants de l'enseignement supérieur au cours de la décennie 1990. Le nombre de diplômés du supérieur a connu une évolution similaire durant cette période. L'une des explications tient à la contraction de l'offre d'emplois qualifiés, elle-même due à la multiplication d'opportunités d'activités dans les services (tourisme). La démotivation de jeunes pour des études longues n'offrant que des perspectives aléatoires d'ascension sociale semble être déterminante pour comprendre 
l'ampleur de cette baisse ${ }^{13}$. Mais alors que le budget de l'éducation supérieure subissait une contraction (- $8 \%$ ) entre 1989 et 1993, sa part dans le PIB continuait de croître sur la période $(+40 \%)$. Dès le début des années 2000 , les taux de scolarisation dans le supérieur s'étaient redressés, et dépassèrent largement ceux de 1989. En 2004, le nombre d'inscrits en cycles supérieurs a été le plus élevé de l'histoire du pays : 380000 étudiants.

La crise a accru le coût d'opportunité de l'éducation et contraint nombre d'étudiants à interrompre leurs études pour chercher du travail, mais elle a aussi exercé une désincitation au travail des enseignants et détourné certains d'entre eux de leur profession. Si la stratégie de réforme éducative est parvenue à ne laisser aucun enseignant sans travail, elle n'a pu éviter que certains exercent un emploi d'appoint ou changent temporairement, ou définitivement, d'activité. Les travailleurs qualifiés (professeurs, médecins, scientifiques) ont été de ceux qui ont le plus migrés vers d'autres secteurs, surtout dans les services, où les qualifications exigées sont souvent bien moins élevées. Mais sur quelque 7 ou $8 \%$ d'enseignants qui avaient cessé d'exercer leur profession en 1993-94, près d'un tiers a réintégré l'éducation nationale dans l'année, traduisant la réversibilité de cette dévalorisation de leurs qualifications. Les revalorisations successives des rémunérations et des compléments et stimulants ne sont pas étrangères à cette évolution. Il n'en reste pas moins que, pendant les temps les plus difficiles de la crise, ces départs de professeurs ont engendre une baisse de l'encadrement - même si le phénomène a été pour partie temporaire et a fini par être résorbé.

L'adoption d'une série de mesures de plein emploi a permis d'améliorer l'appariement entre les formations dispensées et les offres d'emplois liés à la tertiarisation de l'économie ou aux pôles de développement régionaux. Les nouveaux programmes pour l'emploi des années 2000 participent à l'élévation et à la requalification des formations de travailleurs. L'une des innovations les plus décisives en la matière a été de considérer les études comme une forme d'emplois. Le nombre de travailleurs sociaux s'est accru au cours des dernières années, grâce à l'ouverture d'écoles spécialisées. Parallèlement, la «municipalisation » de l'enseignement supérieur, avec l'essor du réseau universitaire au niveau des collectivités locales, a permis de progresser en direction d'une « universalisation de l'université ». En 2004, le pays comptait plus de 700000 diplômés de l'université, parmi lesquels près de 80000 dans des disciplines scientifiques ou technologiques (dont 7000 docteurs ès sciences), et 300000 professeurs de haut niveau, au nombre desquels 69000 enseignent la médecine. C'est donc logiquement que les secteurs scientifiques et leurs pôles d'excellence ont réalisé des progrès unanimement reconnus, tout spécialement dans les biotechnologies, la conception de médicaments et les nouvelles technologies.

La lecture critique que les responsables cubains ont faite de ces réformes éducatives néolibérales les a amené à réaffirmer, malgré les pressions contraires, les principes qui ont guidé les politiques sociales du pays depuis 1959. Cette continuité parait avoir produit des effets positifs, puisque, de l'avis de nombreux experts internationaux, le système éducatif cubain reste, en termes quantitatifs et qualitatifs, l'un des plus performants au monde. À l'évidence, ce constat doit être nuancé, car nombre de problèmes, apparus avec la crise, et sur lesquels nous avons insisté, demeurent à résoudre, faute de quoi la politique éducative de Cuba rencontrerait des difficultés 
pour continuer à réaliser son principe d'égalité et à assurer la correspondance, la complémentarité et la cohérence de ses politiques socio-économiques.

\section{BIBLIOGRAPHIE}

ALHAMA R. (2004) : «Capital Humano, Concepto e instrumentacion », Instituto de Estudios e Investigaciones del Trabajo, La Havane.

BAYART P. (2008) : « Éducation et valorisation à Cuba », Thèse, Université de Paris 1.

CASTAÑEDA R. (2000) : «Consideraciones sobre el nivel y la evolución del Indice de desarrollo humano ", in Cuba in Transition, vol. 10, ASCE 10th Annual Meeting Prodeedings, pp. 234-253, Miami.

CEPAL (2004) : La Economia cubana, Fondo de Cultura económica, Mexico.

CIEM (1996) : Cuba, realidades y potencialidades en el contexto de la integración caribeña, México.

FERRIOL A. (2004) : « Política social y desarrollo : una aproximación global », in E. Alvarez et J. Máttar (eds.), Política social y reformas estructurales : Cuba a principios del siglo XXI, CEPAL-INIEPNUD, juillet, México.

FERRIOL A., RAMOS M. et AÑE L. (2004c) : « ¿Pobreza en la capital ? », INIE, La Havane.

GASPERINI L. (2000) : The Cuban Educa-tion System, Country Studies, World Bank, Washington D.C.

HERRERA R. (dir.) (2006) : Cuba révolutionnaire - Économie et planification, L'Harmattan, Paris.

LAUGLO J. (1996) : «Banking on Education », International Journal of Educational Development, vol. $16, \mathrm{n}^{\circ} 3, \mathrm{pp} .221-233$.

MINED (2002) : La Educación en Cuba, Ministerio de Educación, La Havane.

Ministry of Education (1996) : Cuba Organization of Education, La Havane.

MULOT E. (2001) : « De la Compensation à la réforme sociale $:$ les politiques éducatives de lutte contre la pauvreté au Guatemala », Revue Tiers Monde, vol. XLII, n 168, pp. 885-906.

PNUD (2000) : Investigación sobre desarrollo humano y equidad en Cuba 1999, Caguayo, La Havane. UNESCO (1998) : Primer estudio internacional comparativo en la educación básica, Santiago du Chili.

\section{ANNEXES}


Cuba : données statistiques

\begin{tabular}{|c|c|c|}
\hline Données & Cuba & France \\
\hline Superficie & $110860 \mathrm{~km}^{2}$ & $551500 \mathrm{~km}^{2}$ \\
\hline Population & 11382820 habitants (2005) & $63800000(2008)$ \\
\hline Densité & 102 habitants $/ \mathrm{km}^{2}$ & 107 habitants $/ \mathrm{km}^{2}$ \\
\hline Population urbaine & $75,30 \%$ & $75,60 \%$ \\
\hline Indice fécondité & 1,58 & 1,96 \\
\hline Accroissement population & $0,31 \%$ & $0,46 \%$ \\
\hline Espérance de vie & 76,47 ans & 81,00 ans \\
\hline Jeunes $0-14$ ans & $19,10 \%$ & $18,70 \%$ \\
\hline Nombre d'habitants par médecin & 166 & 333 \\
\hline Lits d'hôpitaux & 6,2 pour 1000 habitants & 8,5 pour 1000 habitants \\
\hline $\begin{array}{l}\text { Indicateur de développement } \\
\text { humain (PNUD) }\end{array}$ & $8 / 10$ (en hausse) & $9 / 10$ (stable) \\
\hline PNB global (milliards de dollars) & 9,98 & 1447,90 \\
\hline PNB par habitant (en dollars) & 892 & 24572 \\
\hline Taux de croissance du PIB & $12,5 \%(2006)$ & $2,00 \%(2006)$ \\
\hline Chômage & $1,9 \%$ & $7,8 \%$ \\
\hline $\begin{array}{l}\text { Part de la dépense intérieure } \\
\text { d'éducation/PIB (2005) }\end{array}$ & $10,10 \%$ & $6,9 \%$ \\
\hline
\end{tabular}

\section{NOTES}

\section{Herrera (2006).}

2. Ferriol (2004).

3. Exemple: M. Machinea, secrétaire exécutif de la CEPAL, de déclarer en 2005 : "Cuba a réalisé un gros effort de formation de "capital humain" et est devenu leader dans l'exportation de services sociaux dans la région ".

4. Mulot (2001).

5. Alhama (2004).

6. Ministry of Education (1996).

7. PNUD (2000).

8. Castañeda (2000).

9. CEPAL (2004).

10. CIEM (1996).

11. UNESCO (1998).

12. Lauglo (1996).

13. Bayart (2008). 
INDEX

Mots-clés : politique éducative, système éducatif

Index géographique : Cuba

\section{AUTEURS}

\section{PHILIPPE BAYART}

Doctorant au Centre d'Économie de la Sorbonne (MATISSE) de l'Université de Paris 1, France.

\section{RÉMY HERRERA}

Chercheur au CNRS, Centre d'Économie de la Sorbonne (MATISSE) de Paris 1, France.

\section{ÉRIC MULOT}

Docteur de l'Université de Paris 1 Panthéon-Sorbonne, chargé d'études, France. 